\title{
A Modified Classification of Prognostic Factors Based on Pathological Stage and Tumor Regression Grade in Patients with Rectal Cancer Who Receive Preoperative Chemoradiotherapy
}

\author{
Toshiyuki Suzuki $^{a}$ Sotaro Sadahiro ${ }^{a}$ Akira Tanaka $^{a}$ Kazutake Okada ${ }^{a}$ \\ Gota Saito $^{a}$ Hiroshi Miyakita $^{a}$ Takeshi Akibab Hiroshi Yamamuro $^{b}$ \\ Departments of a Surgery and ${ }^{b}$ Radiology, Tokai University School of Medicine, Isehara, Japan
}

\section{Keywords}

Rectal cancer · Chemoradiotherapy · Prognostic factor .

Tumor regression grade $\cdot$ ypStage

\section{Abstract}

Objective: The histologic response to neoadjuvant chemoradiotherapy (CRT) has been intimately related to outcomes in locally advanced rectal cancer. However, reliable prognostic factors have yet to be established. Subjects and Methods: The study group comprised 198 patients with locally advanced rectal cancer who received CRT. A modified classification based on the combination of ypStage and tumor regression grade (TRG) was developed. ypStage II with TRG 2 was classified as ypTRGstage IIA, and ypStage II with TRG 3 or 4 was classified as ypTRGstage IIB. ypStage 0 and ypStage I were classified as ypTRGstage I, and ypStage III was classified as ypTRGstage III. Results: The 5-year disease-free survival (DFS) was $83 \%$ in ypTRGstage I, 86\% in ypTRGstage IIA, $57 \%$ in ypTRGstage IIB, and 60\% in ypTRGstage III ( $p=0.0001)$. The 5-year DFS in ypTRGstage IIA did not differ significantly from that in ypStage $0(p=0.865)$ or ypStage I $(p=0.585)$. The 5 -year DFS in ypStage IIB did not differ from that in ypStage III $(p=0.912)$. Multivariate analysis showed that ypTRGstage was an independent risk factor for DFS. Conclusion: A mod-
\end{abstract}

\section{KARGER}

E-Mail karger@karger.com www.karger.com/ocl
(C) 2017 The Author(s)

Open access

This article is licensed under the Creative Commons AttributionUsage and distribution for commercial purposes as well as any distribution of modified material requires written permission.
Published by S. Karger AG, Basel

Karger NonCommercial-NoDerivatives 4.0 International License (CC BYNC-ND) (http://www.karger.com/Services/OpenAccessLicense). ified classification allows patients with ypStage II locally advanced rectal cancer to be clearly divided into two groups: responders and nonresponders.

(C) 2017 The Author(s)

Published by S. Karger AG, Basel

\section{Introduction}

Multidisciplinary treatment including preoperative radiotherapy is the global standard for $\mathrm{T} 3, \mathrm{~N} 0$ or $\mathrm{T}_{\text {any }}$, $\mathrm{N} 1-2$, or T4 rectal cancer and significantly decreases local recurrence [1-4]. However, the European Society for Medical Oncology (ESMO) guidelines [5] claim that clinical T3a or T3b, N0 cancer of the middle or higher rectum has a good outcome and can therefore be treated by surgery alone.

The histologic response to preoperative chemoradiotherapy (CRT) in the radiation field has been reported to be closely related to outcomes. Patients who have posttreatment pathological complete regression, a marked decrease in tumor volume, or downstaging of $\mathrm{T}$ or $\mathrm{N}$ stage have been shown to have significantly better disease-free survival (DFS) and overall survival (OS) than patients without these findings [6-10].

Prof. Sotaro Sadahiro

Department of Surgery, Tokai University School of Medicine 143 Shimokasuya

Isehara, Kanagawa 259-1193 (Japan)

E-Mail sadahiro@is.icc.u-tokai.ac.jp 
However, ypStage II disease includes patients who initially have pT3-T4, N1 disease and show a good response to treatment, resulting in downstaging, patients who initially have pT3-T4, N0 disease who respond to treatment without downstaging, and patients who initially have pT3-T4, N0 disease and show a poor response to treatment. We therefore hypothesized that a new classification more closely related to outcomes could be developed by dividing patients with ypStage II disease into two groups: those with a good histologic response and those with a poor histologic response. We thus designed a modified classification based on the posttreatment pathological stage (ypStage) and tumor regression grade (TRG).

\section{Subjects and Methods}

Between 2007 and 2015, total mesorectal excision surgery combined with preoperative CRT was used to treat 198 patients with histologically proven adenocarcinoma of the middle or lower rectum who had a preoperative diagnosis of clinical stage (cStage) II or stage III disease according to the TNM classification.

The initial evaluation included digital examination of the rectum, colonoscopy, barium enema, computed tomography of the chest, abdomen, and pelvis, endorectal ultrasonography, and magnetic resonance imaging of the pelvis.

\section{Chemoradiotherapy}

Preoperative radiotherapy was performed with 18-MV X-ray beams delivered by a linear accelerator (Clinac 2100C; Varian Medical Systems, Inc., Palo Alto, CA, USA) using a four-field technique. Irradiation was performed once ( 1.8 or $2 \mathrm{~Gy}$ ) daily to a total dose of 40-45 Gy. Total mesorectal excision surgery was done 6-8 weeks (range, 30-65 days) after the completion of irradiation.

Concomitant chemotherapy with oral uracil-tegafur $(400 \mathrm{mg} /$ $\left.\mathrm{m}^{2}\right)$ or oral S-1 $\left(80 \mathrm{mg} / \mathrm{m}^{2}\right)$ was started at the same time as radiotherapy. Oral uracil-tegafur was simultaneously given with radiotherapy on 5 weekdays, followed by a 2 -day rest on weekends. This cycle was repeated during irradiation. Oral S-1 $\left(80 \mathrm{mg} / \mathrm{m}^{2}\right)$ was simultaneously started with radiotherapy. S-1 was given for 2 consecutive weeks, followed by a 1-week rest, and was then given for another 2 weeks $[11,12]$.

Histologic regression was assessed using the Mandard TRG [13] as follows: TRG 1, complete regression; TRG 2, presence of rare residual cancer cells; TRG 3 , fibrosis outgrowing residual cancer cells; TRG 4, residual cancer cells outgrowing fibrosis; and TRG 5 , absence of regression.

We designed a modified classification based on the TRG that classified patients with ypStage II disease into two groups. Patients with TRG 2 were classified as ypTRGstage IIA, and those with TRG 3 or 4 were classified as ypTRGstage IIB (Table 1).

\section{Statistical Analysis}

Statistical analysis was performed using SPSS for Windows, version 23 (IBM Japan, Ltd., Tokyo, Japan). Actuarial survival curves were calculated according to the Kaplan-Meier method. The probabilities of DFS and OS were determined by log-rank
Table 1. Modified prognostic classification consisting of ypStage and TRG

\begin{tabular}{ll}
\hline ypTRGstage I & ypStage 0, I \\
ypTRGstage IIA & ypStage II + TRG 2 \\
ypTRGstage IIB & ypStage II + TRG 3, 4 \\
ypTRGstage III & ypStage III
\end{tabular}

TRG, tumor regression grade.

analysis. A Cox proportional hazards model was used to adjust comparisons for clinicopathological variables. $p$ values $<0.05$ were considered to indicate statistical significance.

\section{Results}

\section{Patient and Tumor Characteristics}

Table 2 shows the patient characteristics. There were 150 men and 48 women with a median age of 64 years. Overall, 85 patients had clinical stage II disease and 113 had clinical stage III disease. The chemotherapeutic drugs administered concurrently with preoperative radiotherapy were S-1 in 153 patients $(77.3 \%)$ and uraciltegafur in 45 patients $(22.7 \%)$. Sphincter-preserving surgery was performed in 147 patients (74.2\%). Before CRT, $96.5 \%$ of the patients had clinical T3 or T4 disease, and $57.1 \%$ had clinical N1 or cN2 disease. After CRT, however, the proportion of patients with pathological T3 or $\mathrm{T} 4$ disease decreased to $51.5 \%$, and the proportion of patients with pathological $\mathrm{N} 1$ or $\mathrm{N} 2$ disease decreased to $22.7 \%$.

Pathological complete response (pCR) was found in 31 patients (15.7\%). One patient had complete regression (TRG 1) of the primary lesion, but was pathologically node-positive $(\mathrm{pN}+)$. Among the 40 patients with pathological T0 disease, 8 had carcinoma in situ (Tis).

The TRG was TRG 1 in 32 patients (16.2\%), TRG 2 in 52 patients $(26.3 \%)$, TRG 3 in 78 patients $(39.4 \%)$, and TRG 4 in 36 patients (18.2\%). No patient had TRG 5. The ypStage was ypStage 0 in 39 patients (19.7\%), ypStage I in 49 patients $(24.7 \%)$, ypStage II in 65 patients $(32.8 \%)$, and ypStage III in 45 patients (22.7\%). Overall, 88 patients had ypTRGstage I, consisting of ypStage 0 and I combined. The 65 patients with ypStage II were classified into $16 \mathrm{pa}-$ tients with ypTRGstage IIA and 49 patients with ypTRGstage IIB. Forty-five patients had ypTRGstage III, which was identical to ypStage III.
Oncology 2017;93:287-294 DOI: $10.1159 / 000478266$
Suzuki/Sadahiro/Tanaka/Okada/Saito/ Miyakita/Akiba/Yamamuro 
Table 2. Patient characteristics

\begin{tabular}{|c|c|c|c|}
\hline & $\begin{array}{l}n(\%) \text { or } \\
\text { median (range) }\end{array}$ & & $\begin{array}{l}n(\%) \text { or } \\
\text { median (range) }\end{array}$ \\
\hline Gender & & урТ & \\
\hline Male & $150(75.8 \%)$ & 0 & $40(20.2 \%)$ \\
\hline Female & $48(24.2 \%)$ & 1 & $13(6.6 \%)$ \\
\hline Age, years & $64(32-92)$ & 2 & $43(21.7 \%)$ \\
\hline Tumor location & & 3 & $98(49.5 \%)$ \\
\hline Middle rectum & $78(39.4 \%)$ & 4 & $4(2.0 \%)$ \\
\hline Lower rectum & $120(60.6 \%)$ & ypN & \\
\hline Histologic type & & 0 & $153(77.3 \%)$ \\
\hline Well & $144(72.7 \%)$ & 1 & $31(15.7 \%)$ \\
\hline Moderate & $52(26.3 \%)$ & 2 & $14(7.0 \%)$ \\
\hline Poor & $2(1.0 \%)$ & Lymphatic invasion & \\
\hline Clinical T stage & & $(-)$ & $135(68.2 \%)$ \\
\hline 2 & $7(3.5 \%)$ & $(+)$ & $63(31.8 \%)$ \\
\hline 3 & $159(80.3 \%)$ & Venous invasion & \\
\hline 4 & $32(16.2 \%)$ & $(-)$ & $120(60.6 \%)$ \\
\hline Clinical $\mathrm{N}$ stage & & $(+)$ & $78(39.4 \%)$ \\
\hline 0 & $85(42.9 \%)$ & TRG & \\
\hline 1 & $73(36.9 \%)$ & 1 & $32(16.2 \%)$ \\
\hline 2 & $40(20.2 \%)$ & 2 & $52(26.3 \%)$ \\
\hline Clinical stage & & 3 & $78(39.4 \%)$ \\
\hline II & $85(42.9 \%)$ & 4 & $36(18.2 \%)$ \\
\hline III & $113(57.1 \%)$ & 5 & $0(0 \%)$ \\
\hline Radiation dose & & ypStage & \\
\hline $40 \mathrm{~Gy}$ & $14(7.1 \%)$ & 0 & $39(19.7 \%)$ \\
\hline $45 \mathrm{~Gy}$ & $184(92.9 \%)$ & I & $49(24.7 \%)$ \\
\hline Concurrent chemotherapy & & II & $65(32.8 \%)$ \\
\hline Uracil-tegafur & $45(22.7 \%)$ & III & $45(22.7 \%)$ \\
\hline S-1 & $153(77.3 \%)$ & ypTRGstage & \\
\hline Surgical procedure & & I & $88(44.4 \%)$ \\
\hline Low anterior resection & $147(74.2 \%)$ & IIA & $16(8.1 \%)$ \\
\hline Abdominoperineal resection & $51(25.8 \%)$ & IIB & $49(24.7 \%)$ \\
\hline Adjuvant chemotherapy & & III & $45(22.7 \%)$ \\
\hline Administered & $117(59.1 \%)$ & & \\
\hline Not administered & $81(40.9 \%)$ & & \\
\hline
\end{tabular}

Lower rectum, lower third of the rectum; middle rectum, middle third of the rectum; TRG, tumor regression grade.

\section{Survival Analysis}

The median follow-up was 52 months (range, 1-168). The 5 -year DFS was $71 \%$, and the 5 -year OS was $85 \%$. Recurrence was found in 48 patients $(24.2 \%)$. The initial site of recurrence was locoregional in 6 patients (3.0\%), the liver in 17 patients (8.6\%), the lung in 19 patients (9.6\%), and distant lymph nodes in 6 patients (3.0\%).

The 5-year DFS rate according to the TRG was as follows: TRG 1, 89\%; TRG 2, 76\%; TRG 3, 67\%; and TRG 4, $59 \%$. The 5-year OS rate according to the TRG was as follows: TRG 1, 96\%; TRG 2, 82\%; TRG 3, 84\%; and TRG 4, $83 \%$. Marked tumor regression (TRG 1 or 2 ) was con-

Prognostic Factors Based on ypStage and Tumor Regression Grade trasted with TRG 3 or 4 . The DFS rate was significantly higher in patients with TRG 1 or 2 than in those with TRG 3 or $4(p=0.014)$. The OS rate did not differ between these groups $(p=0.744)$.

The 5-year DFS rate according to the ypStage was as follows: ypStage 0, 88\%; ypStage I, 78\%; ypStage II, 64\%; and ypStage III, $60 \%(p=0.002)$ (Fig. 1a). The 5-year OS rate according to ypStage was as follows: ypStage 0, 97\%; ypStage I, 86\%; ypStage II, 83\%; and ypStage III, $80 \%$ $(p=0.220)$ (Fig. 1b).

The 5-year DFS rate according to the ypTRGstage was as follows: ypTRGstage I, 83\%; ypTRGstage IIA,

Oncology 2017;93:287-294 289 


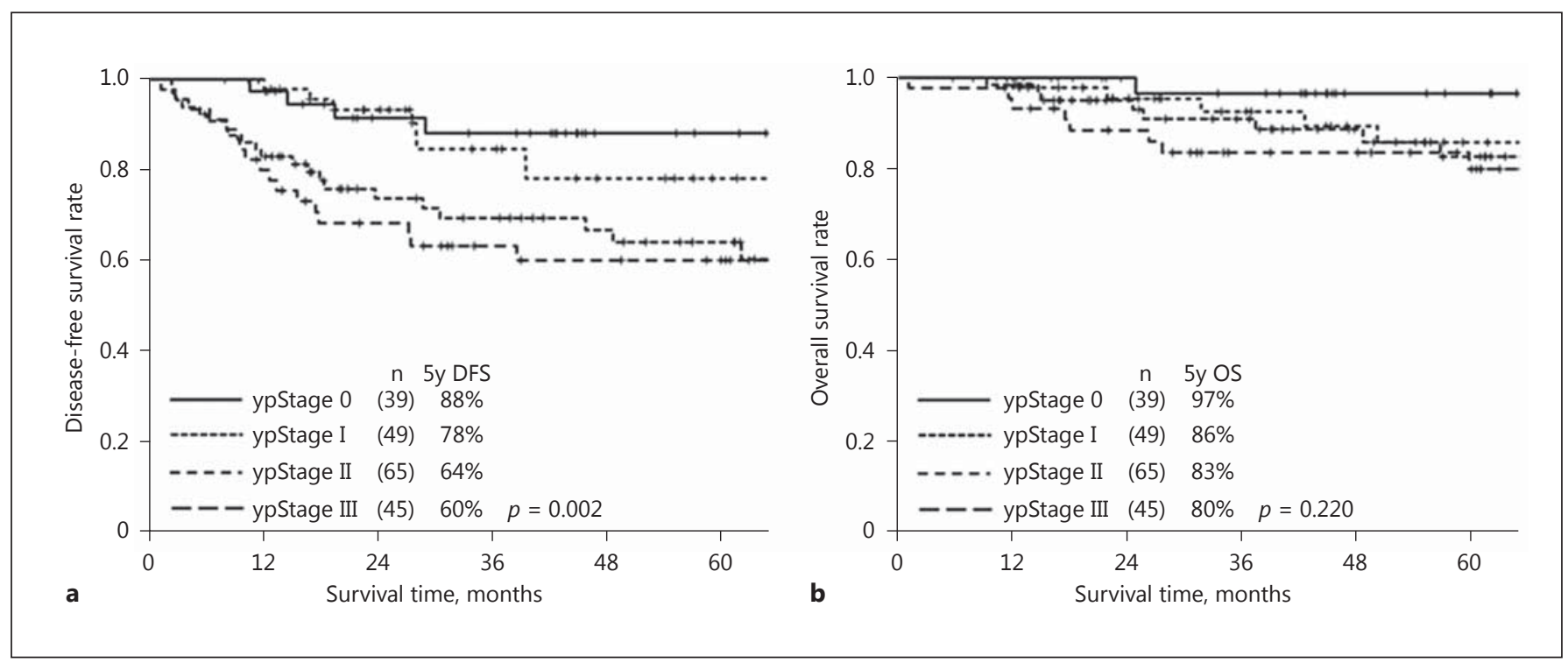

Fig. 1. Disease-free survival (a) and overall survival (b) according to ypStage. 5y DFS, 5-year disease-free survival; 5y OS, 5-year overall survival.

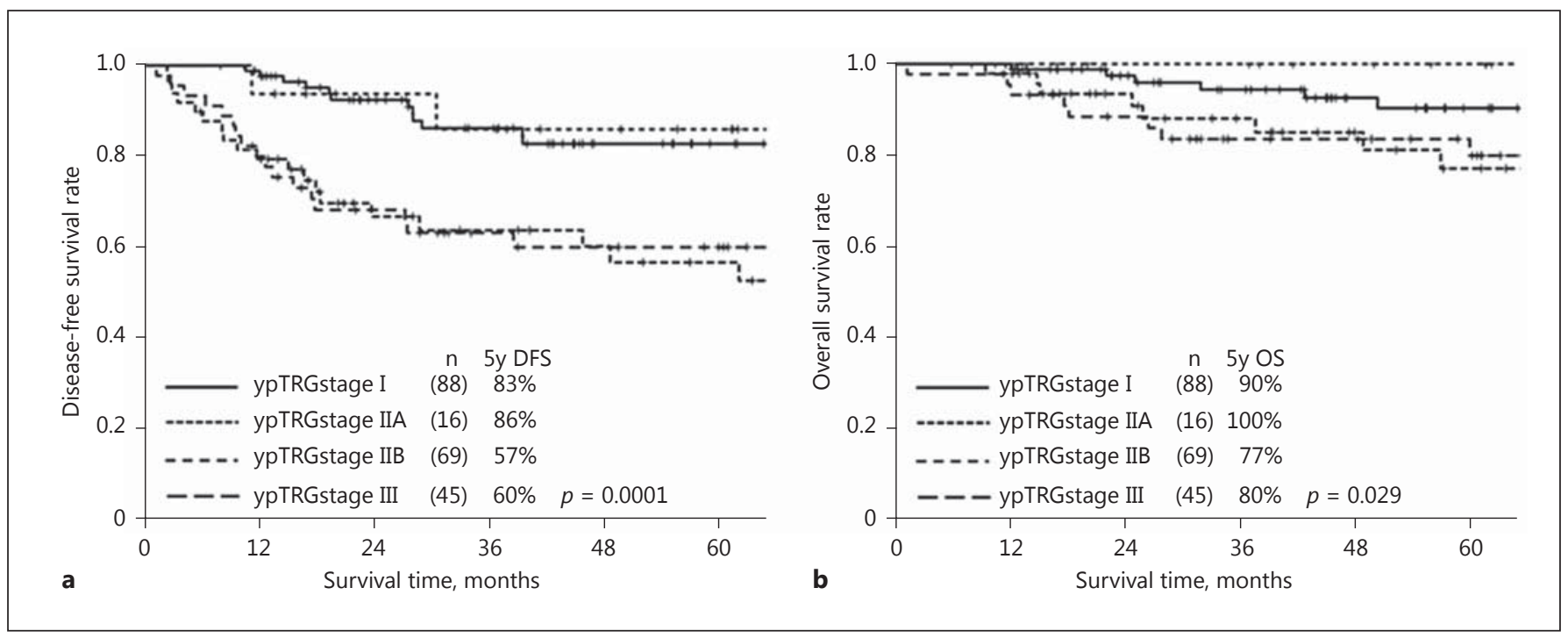

Fig. 2. Disease-free survival (a) and overall survival (b) according to ypTRGstage. 5y DFS, 5-year disease-free survival; 5y OS, 5-year overall survival.

86\%; ypTRGstage IIB, 57\%; and ypTRGstage III, 60\% ( $p=0.0001$ ) (Fig. 2a). The 5-year OS rate according to the ypTRGstage was as follows: ypTRGstage I, 90\%; ypTRGstage IIA, 100\%; ypTRGstage IIB, 77\%; and ypTRGstage III, $80 \%$ ( $p=0.029$ ) (Fig. $2 b$ ).

When ypStage was compared with ypTRGstage, the 5 -year DFS rate in ypTRGstage IIA did not differ from that in ypStage $0(p=0.865)$ or ypStage I ( $p=0.585)$, and the 5-year DFS rate in ypTRGstage IIB did not differ from that in ypStage III $(p=0.912)$. Similarly, the 5 -year OS rate in ypTRGstage IIA did not differ from that in ypStage $0(p=0.520)$ or ypStage I $(p=0.179)$, and the 5 -year OS rate in ypTRGstage IIB did not differ from that in ypStage III $(p=0.595)$. 
The 65 patients with ypStage II, comprising the largest subgroup of patients according to ypStage, could be classified into 16 patients with ypTRGstage IIA and a good prognosis and 49 patients with ypTRGstage IIB and a poor prognosis.

\section{Prognostic Factors Affecting Survival}

TRG 1 and 2, ypStage 0 and I, and ypTRGstage 0 , I, and IIA were collectively considered to indicate a good response. Cox univariate analysis was performed to assess the relations of these variables to DFS. DFS correlated with ypT, post-CRT pathological nodal status (ypN), lymphatic invasion, venous invasion, TRG, ypStage, and ypTRGstage (Table 3).

Significant factors in the Cox univariate analysis were included in multivariate analysis. The results showed that ypT, ypN, and ypStage correlated with each other. Since ypT, ypN, TRG, and ypTRGstage correlated with each other, three types of multivariate analyses were performed (Table 4). The results showed that ypT, ypStage, and ypTRGstage were independent risk factors for DFS. The hazard ratio of ypTRGstage was 2.876 (95\% confidence interval, 1.510-5.477), which was greater than the hazard ratio of ypStage $(2.274$; $95 \%$ confidence interval, 1.152 4.488).

Cox univariate analysis showed that OS was related to ypT, lymphatic invasion, ypStage, and ypTRGstage. Multivariate analysis showed that lymphatic invasion was an independent risk factor for OS (Table 5).

\section{Discussion}

Previous studies have reported that post-CRT ypT, ypN, ypStage, and histologic response (TRG) are related to oncologic outcomes $[6,8,10,14-21]$. A four-level subclassification of the depth of extramuscular invasion has been proposed for pathological T stage (pT stage) [22]. In patients who are treated by surgery alone, DFS differs according to the subclassification of $\mathrm{pT}$, but does not differ in patients who have received preoperative CRT. Therefore, $\mathrm{pT}$ has been reported not to be a prognostic factor $[23,24]$.

The TRG has been widely used to evaluate the histologic response to preoperative CRT. Quah et al. [25] reported that ypStage was more strongly associated with DFS than was TRG in 342 patients with rectal cancer who received preoperative CRT.

Patients with pCR $[6,8,16,18,26-28]$ and those with good tumor regression even in the absence of pCR $[8,16$,

Prognostic Factors Based on ypStage and Tumor Regression Grade
Table 3. Univariate Cox proportional hazards regression model of clinical factors of disease-free survival

\begin{tabular}{|c|c|c|c|c|}
\hline & $\begin{array}{l}n(\%) \text { or } \\
\text { median (range) }\end{array}$ & $\begin{array}{l}p \\
\text { value }\end{array}$ & HR & $95 \% \mathrm{CI}$ \\
\hline \multicolumn{5}{|l|}{ Gender } \\
\hline Male & $150(75.8 \%)$ & & 1 & \\
\hline Female & $48(24.2 \%)$ & 0.654 & 1.164 & $0.599-2.263$ \\
\hline Age, years & $64(32-92)$ & 0.865 & 0.951 & $0.533-1.695$ \\
\hline \multicolumn{5}{|l|}{ Tumor location } \\
\hline Middle rectum & $78(39.4 \%)$ & & 1 & \\
\hline Lower rectum & $120(60.6 \%)$ & 0.196 & 1.459 & $0.823-2.588$ \\
\hline \multicolumn{5}{|l|}{ Histologic type } \\
\hline Well & $144(72.7 \%)$ & & 1 & \\
\hline Moderate/poor & $54(27.3 \%)$ & 0.155 & 1.497 & $0.858-2.610$ \\
\hline \multicolumn{5}{|l|}{ Clinical T stage } \\
\hline 2 & $7(3.5 \%)$ & & 1 & \\
\hline 3 & $159(80.3 \%)$ & 0.667 & 0.732 & $0.177-3.031$ \\
\hline 4 & $32(16.2 \%)$ & 0.818 & 0.836 & $0.182-3.834$ \\
\hline \multicolumn{5}{|l|}{ Clinical N stage } \\
\hline$(-)$ & $85(42.9 \%)$ & & 1 & \\
\hline$(+)$ & $113(57.1 \%)$ & 0.958 & 1.014 & $0.592-1.738$ \\
\hline \multicolumn{5}{|l|}{ Clinical stage } \\
\hline II & $85(42.9 \%)$ & & 1 & \\
\hline III & $113(57.1 \%)$ & 0.958 & 1.014 & $0.592-1.738$ \\
\hline \multicolumn{5}{|l|}{ Radiation dose } \\
\hline $40 \mathrm{~Gy}$ & $14(7.1 \%)$ & & 1 & \\
\hline $45 \mathrm{~Gy}$ & $184(92.9 \%)$ & 0.105 & 0.527 & $0.244-1.142$ \\
\hline \multicolumn{5}{|c|}{ Concurrent chemotherapy } \\
\hline Uracil-tegafur & $45(22.7 \%)$ & & 1 & \\
\hline S-1 & $153(77.3 \%)$ & 0.673 & 0.870 & $0.457-1.658$ \\
\hline \multicolumn{5}{|l|}{ ypT } \\
\hline $0,1,2$ & $96(48.5 \%)$ & & 1 & \\
\hline 3,4 & $102(51.5 \%)$ & $<0.001$ & 3.329 & $1.820-6.089$ \\
\hline \multicolumn{5}{|l|}{ ypN } \\
\hline$(-)$ & $153(77.3 \%)$ & & 1 & \\
\hline$(+)$ & $45(22.7 \%)$ & 0.012 & 2.048 & $1.173-3.578$ \\
\hline \multicolumn{5}{|l|}{ Lymphatic invasion } \\
\hline$(-)$ & $135(68.2 \%)$ & & 1 & \\
\hline$(+)$ & $63(31.8 \%)$ & 0.001 & 2.511 & $1.478-4.266$ \\
\hline \multicolumn{5}{|l|}{ Venous invasion } \\
\hline$(-)$ & $120(60.6 \%)$ & & 1 & \\
\hline$(+)$ & $78(39.4 \%)$ & 0.014 & 1.946 & $1.144-3.310$ \\
\hline \multicolumn{5}{|l|}{ TRG } \\
\hline 1,2 & $84(42.4 \%)$ & & 1 & \\
\hline $3,4,5$ & $114(57.6 \%)$ & 0.016 & 2.081 & $1.148-3.772$ \\
\hline \multicolumn{5}{|l|}{ ypStage } \\
\hline $0 / \mathrm{I}$ & $88(44.4 \%)$ & & 1 & \\
\hline II/III & $110(55.6 \%)$ & $<0.001$ & 3.069 & $1.651-5.704$ \\
\hline \multicolumn{5}{|l|}{ ypTRGstage } \\
\hline I/IIA & $104(52.5 \%)$ & & 1 & \\
\hline IIB/III & $94(47.5 \%)$ & $<0.001$ & 3.590 & $1.989-6.481$ \\
\hline
\end{tabular}

CI, confidence interval; HR, hazard ratio; lower rectum, lower third of the rectum; middle rectum, middle third of the rectum; TRG, tumor regression grade.

$17,21,26,28-31]$ have been reported to have good outcomes. Agarwal et al. [28] studied 251 patients with locally advanced rectal adenocarcinoma who received preoperative CRT and found that recurrence-free survival

Oncology 2017;93:287-294 291 
Table 4. Multivariate Cox proportional hazards regression model of clinical factors of disease-free survival

\begin{tabular}{|c|c|c|c|c|}
\hline & $n(\%)$ & $p$ value & HR & $95 \% \mathrm{CI}$ \\
\hline \multicolumn{5}{|l|}{ урт } \\
\hline $0,1,2$ & $96(48.5 \%)$ & & 1 & \\
\hline 3,4 & $102(51.5 \%)$ & 0.008 & 2.511 & $1.278-4.933$ \\
\hline \multicolumn{5}{|l|}{ ypN } \\
\hline$(-)$ & $153(77.3 \%)$ & & 1 & \\
\hline$(+)$ & $45(22.7 \%)$ & 0.797 & 1.089 & $0.568-2.086$ \\
\hline \multicolumn{5}{|c|}{ Lymphatic invasion } \\
\hline$(-)$ & $135(68.2 \%)$ & & 1 & \\
\hline$(+)$ & $63(31.8 \%)$ & 0.134 & 1.648 & $0.858-3.168$ \\
\hline \multicolumn{5}{|c|}{ Venous invasion } \\
\hline$(-)$ & $120(60.6 \%)$ & & 1 & \\
\hline$(+)$ & $78(39.4 \%)$ & 0.873 & 1.052 & $0.561-1.974$ \\
\hline \multicolumn{5}{|l|}{ TRG } \\
\hline 1,2 & $84(42.4 \%)$ & & 1 & \\
\hline $3,4,5$ & $114(57.6 \%)$ & 0.612 & 1.190 & $0.607-2.334$ \\
\hline \multicolumn{5}{|c|}{ Lymphatic invasion } \\
\hline$(-)$ & $135(68.2 \%)$ & & 1 & \\
\hline$(+)$ & $63(31.8 \%)$ & 0.084 & 1.707 & $0.931-3.131$ \\
\hline \multicolumn{5}{|c|}{ Venous invasion } \\
\hline$(-)$ & $120(60.6 \%)$ & & 1 & \\
\hline$(+)$ & $78(39.4 \%)$ & 0.753 & 1.104 & $0.596-2.045$ \\
\hline \multicolumn{5}{|l|}{ TRG } \\
\hline 1,2 & $84(42.4 \%)$ & & 1 & \\
\hline $3,4,5$ & $114(57.6 \%)$ & 0.593 & 1.201 & $0.613-2.353$ \\
\hline \multicolumn{5}{|l|}{ ypStage } \\
\hline $0 / \mathrm{I}$ & $88(44.4 \%)$ & & 1 & \\
\hline II/III & $110(55.6 \%)$ & 0.018 & 2.274 & $1.152-4.488$ \\
\hline \multicolumn{5}{|c|}{ Lymphatic invasion } \\
\hline$(-)$ & $135(68.2 \%)$ & & 1 & \\
\hline$(+)$ & $63(31.8 \%)$ & 0.116 & 1.614 & $0.888-2.933$ \\
\hline \multicolumn{5}{|c|}{ Venous invasion } \\
\hline$(-)$ & $120(60.6 \%)$ & & 1 & \\
\hline$(+)$ & $78(39.4 \%)$ & 0.736 & 1.107 & $0.614-1.995$ \\
\hline \multicolumn{5}{|l|}{ ypTRGstage } \\
\hline I/IIA & $104(52.5 \%)$ & & 1 & \\
\hline IIB/III & $94(47.5 \%)$ & 0.001 & 2.876 & $1.510-5.477$ \\
\hline
\end{tabular}

CI, confidence interval; HR, hazard ratio; TRG, tumor regression grade.

did not differ between patients with pCR and those with nearly complete response (residual cancer cells $\leq 5 \%$ ). Guillem et al. [8] studied 297 patients with rectal cancer who received preoperative CRT and reported that the survival rate was significantly higher in patients with $\mathrm{pCR}$ or nearly pCR ( $\geq 95 \%$ tumor response) than in patients with other responses.

On the basis of these results, evaluation methods combining ypStage and TRG have been studied to identify better prognostic factors. Cui et al. [30] studied 329 patients with clinical stage II or III rectal adenocarcinoma who received preoperative CRT and developed a modified T stage classification metric (M-TTRG) that catego-
Table 5. Disease-free survival according to TRG, ypStage and ypTRGstage

\begin{tabular}{llll}
\hline & $\begin{array}{l}\text { 1-year } \\
\text { DFS }\end{array}$ & $\begin{array}{l}\text { 3-year } \\
\text { DFS }\end{array}$ & $\begin{array}{l}5 \text {-year } \\
\text { DFS }\end{array}$ \\
\hline TRG & & & \\
$\quad 1,2$ & $94 \%$ & $85 \%$ & $81 \%$ \\
$\quad 3,4,5$ & $86 \%$ & $69 \%$ & $64 \%$ \\
ypStage & & & \\
$\quad$ 0/I & $99 \%$ & $86 \%$ & $83 \%$ \\
$\quad$ II/III & $82 \%$ & $67 \%$ & $62 \%$ \\
ypTRGstage & & & $83 \%$ \\
$\quad$ I/IIA & $98 \%$ & $86 \%$ & $89 \%$ \\
IIB/III & $80 \%$ & $64 \%$ & $59 \%$ \\
\hline
\end{tabular}

DFS, disease-free survival; TRG, tumor regression grade.

rized patients into five subgroups on the basis of ypT stage and TRG. This system was combined with ypN stage to develop a modified ypTNM staging system that categorizes patients into seven subgroups. However, this system is very complex.

Some patients with ypStage II histologically have a nearly pCR without downstaging owing to the presence of small amounts of cancer cells in extramuscular tissue. The ypTRGstage that we proposed allowed patients with ypStage II to be classified into two subgroups: patients with ypStage II who have a good response to treatment with no downstaging and patients with ypStage II who have a poor response to treatment. This classification of responders and nonresponders was more closely related to outcomes.

In patients who have received neoadjuvant chemotherapy, the indications for and regimens of postoperative adjuvant chemotherapy remain controversial [32, 33 . In the present study, the rate of adjuvant chemotherapy was $44 \%$ in ypStage 0 or I disease, $60 \%$ in ypStage II or III disease, $45 \%$ in ypTRGstage I or IIA disease, and $62 \%$ in ypTRGstage IIB or III disease. The rates of adjuvant chemotherapy were significantly higher in ypStage II or III disease $(p=0.040)$ and in ypTRGstage IIB or III disease $(p=0.029)$.

Our study was retrospective and has several important limitations. It was performed in a single hospital and included only 198 patients. Further multicenter studies of larger number of patients are needed to verify our results.

The ypTRGstage consists of four classes. The survival rate did not differ between ypTRGstage I and IIA, associated with a good response, or between ypTRGstage IIB and III, associated with a poor response. Further studies are therefore needed to determine whether patients who
Oncology 2017;93:287-294
DOI: $10.1159 / 000478266$
Suzuki/Sadahiro/Tanaka/Okada/Saito/ Miyakita/Akiba/Yamamuro 
have ypTRGstage I and IIA can be simply classified as responders and those with ypTRGstage IIB and III as nonresponders.

\section{Conclusions}

A modified classification based on the combination of ypStage and TRG as prognostic factors allowed patients with ypStage II locally advanced rectal cancer who received neoadjuvant CRT to be classified into good and poor responders. This classification is a useful straightforward method for predicting outcomes.

\section{Statement of Ethics}

This study was approved by the institutional review board of Tokai University (08R-032), and all patients provided written informed consent.

\section{Disclosure Statement}

The authors report no potential conflicts of interest.

\section{References}

1 Bosset JF, Collette L, Calais G, Mineur L, Maingon P, Radosevic-Jelic L, Daban A, Bardet E, Beny A, Ollier JC: Chemotherapy with preoperative radiotherapy in rectal cancer. $\mathrm{N}$ Engl J Med 2006;355:1114-1123.

2 Peeters KC, Marijnen CA, Nagtegaal ID, Kranenbarg EK, Putter H, Wiggers T, Rutten H, Pahlman L, Glimelius B, Leer JW, van de Velde CJ: The TME trial after a median follow-up of 6 years: increased local control but no survival benefit in irradiated patients with resectable rectal carcinoma. Ann Surg 2007; 246:693-701.

3 Sauer R, Liersch T, Merkel S, Fietkau R, Hohenberger W, Hess C, Becker H, Raab HR, Villanueva MT, Witzigmann H, Wittekind C, Beissbarth T, Rodel C: Preoperative versus postoperative chemoradiotherapy for locally advanced rectal cancer: results of the German CAO/ARO/AIO-94 randomized phase III trial after a median follow-up of 11 years. J Clin Oncol 2012;30:1926-1933.

4 National Comprehensive Cancer Network: Clinical Practice Guidelines in Oncology. Rectal Cancer v.3. 2017. https://www.nccn. org/professionals/physician_gls/pdf/rectal. pdf (accessed July 1, 2017).

5 Glimelius B, Tiret E, Cervantes A, Arnold D; ESMO Guidelines Working Group: Rectal cancer: ESMO Clinical Practice Guidelines for diagnosis, treatment and follow-up. Ann Oncol 2013;24(suppl 6):vi81-vi88.

6 Maas M, Nelemans PJ, Valentini V, Das P, Rodel C, Kuo LJ, Calvo FA, Garcia-Aguilar J, Glynne-Jones R, Haustermans K, Mohiuddin M, Pucciarelli S, Small W Jr, Suarez J, Theodoropoulos G, Biondo S, Beets-Tan RG, Beets GL: Long-term outcome in patients with a pathological complete response after chemoradiation for rectal cancer: a pooled analysis of individual patient data. Lancet Oncol 2010; 11:835-844.
7 Chapet O, Gerard JP, Riche B, Alessio A, Mornex F, Romestaing P: Prognostic value of tumor regression evaluated after first course of radiotherapy for anal canal cancer. Int $\mathrm{Ja}$ diat Oncol Biol Phys 2005;63:1316-1324.

8 Guillem JG, Chessin DB, Cohen AM, Shia J, Mazumdar M, Enker W, Paty PB, Weiser MR, Klimstra D, Saltz L, Minsky BD, Wong WD: Long-term oncologic outcome following preoperative combined modality therapy and total mesorectal excision of locally advanced rectal cancer. Ann Surg 2005;241:829-836; discussion 836-838.

9 Wolthuis AM, Penninckx F, Haustermans K, Ectors N, Van Cutsem E, D'Hoore A: Outcome standards for an organ preservation strategy in stage II and III rectal adenocarcinoma after neoadjuvant chemoradiation. Ann Surg Oncol 2011;18:684-690.

10 de Campos-Lobato LF, Stocchi L, da Luz Moreira A, Kalady MF, Geisler D, Dietz D, Lavery IC, Remzi FH, Fazio VW: Downstaging without complete pathologic response after neoadjuvant treatment improves cancer outcomes for cIII but not cII rectal cancers. Ann Surg Oncol 2010;17:1758-1766.

11 Sadahiro S, Suzuki T, Tanaka A, Okada K, Kamijo A, Murayama C, Akiba T, Nakayama Y: Phase I/II study of preoperative concurrent chemoradiotherapy with S-1 for locally advanced, resectable rectal adenocarcinoma. Oncology 2011;81:306-311.

12 Suzuki T, Sadahiro S, Tanaka A, Okada K, Kamata H, Kamijo A, Murayama C, Akiba T, Kawada S: Biopsy specimens obtained 7 days after starting chemoradiotherapy (CRT) provide reliable predictors of response to CRT for rectal cancer. Int J Radiat Oncol Biol Phys 2013;85:1232-1238.
13 Mandard AM, Dalibard F, Mandard JC, Marnay J, Henry-Amar M, Petiot JF, Roussel A, Jacob JH, Segol P, Samama G, et al: Pathologic assessment of tumor regression after preoperative chemoradiotherapy of esophageal carcinoma. Clinicopathologic correlations. Cancer 1994;73:2680-2686.

14 Collette L, Bosset JF, den Dulk M, Nguyen F, Mineur L, Maingon P, Radosevic-Jelic L, Pierart M, Calais G: Patients with curative resection of cT3-4 rectal cancer after preoperative radiotherapy or radiochemotherapy: does anybody benefit from adjuvant fluorouracilbased chemotherapy? A trial of the European Organisation for Research and Treatment of Cancer Radiation Oncology Group. J Clin Oncol 2007;25:4379-4386.

15 Kulaylat AS, Hollenbeak CS, Stewart DB Sr: Adjuvant chemotherapy improves overall survival of rectal cancer patients treated with neoadjuvant chemoradiotherapy regardless of pathologic nodal status. Ann Surg Oncol 2017;24:1281-1288.

16 Zhang LN, Xiao WW, Xi SY, OuYang PY, You KY, Zeng ZF, Ding PR, Zhang HZ, Pan $\mathrm{ZZ}$, Xu RH, Gao YH: Pathological assessment of the AJCC tumor regression grading system after preoperative chemoradiotherapy for Chinese locally advanced rectal cancer. Medicine (Baltimore) 2016;95:e2272.

17 Fokas E, Liersch T, Fietkau R, Hohenberger W, Beissbarth T, Hess C, Becker H, Ghadimi M, Mrak K, Merkel S, Raab HR, Sauer R, Wittekind C, Rodel C: Tumor regression grading after preoperative chemoradiotherapy for locally advanced rectal carcinoma revisited: updated results of the CAO/ARO/AIO-94 trial. J Clin Oncol 2014;32:1554-1562.

18 Kuo LJ, Liu MC, Jian JJ, Horng CF, Cheng TI, Chen CM, Fang WT, Chung YL: Is final TNM staging a predictor for survival in locally advanced rectal cancer after preoperative chemoradiation therapy? Ann Surg Oncol 2007;14:2766-2772. 
19 Kim NK, Baik SH, Seong JS, Kim H, Roh JK, Lee KY, Sohn SK, Cho CH: Oncologic outcomes after neoadjuvant chemoradiation followed by curative resection with tumor-specific mesorectal excision for fixed locally advanced rectal cancer: impact of postirradiated pathologic downstaging on local recurrence and survival. Ann Surg 2006;244:1024-1030.

20 Belluco C, Forlin M, Olivieri M, Cannizzaro R, Canzonieri V, Buonadonna A, Bidoli E, Matrone F, Bertola G, De Paoli A: Long-term outcome of rectal cancer with clinically (EUS/ MRI) metastatic mesorectal lymph nodes treated by neoadjuvant chemoradiation: role of organ preservation strategies in relation to pathologic response. Ann Surg Oncol 2016; 23:4302-4309.

21 Rodel C, Martus P, Papadoupolos T, Fuzesi L, Klimpfinger M, Fietkau R, Liersch T, Hohenberger W, Raab R, Sauer R, Wittekind C: Prognostic significance of tumor regression after preoperative chemoradiotherapy for rectal cancer. J Clin Oncol 2005;23:86888696.

22 Compton CC, Greene FL: The staging of colorectal cancer: 2004 and beyond. CA Cancer J Clin 2004;54:295-308.

23 Zinicola R, Pedrazzi G, Haboubi N, Nicholls $\mathrm{RJ}$ : The degree of extramural spread of T3 rectal cancer: an appeal to the American Joint Committee on Cancer. Colorectal Dis 2017; 19:8-15.

24 Ogura A, Akiyoshi T, Yamamoto N, Kawachi H, Ishikawa Y, Noma H, Nagino M, Fukunaga Y, Ueno M: Does the depth of mesorectal invasion have prognostic significance in patients with ypT3 lower rectal cancer treated with preoperative chemoradiotherapy? Int J Colorectal Dis 2017;32:349-356.

25 Quah HM, Chou JF, Gonen M, Shia J, Schrag D, Saltz LB, Goodman KA, Minsky BD, Wong WD, Weiser MR: Pathologic stage is most prognostic of disease-free survival in locally advanced rectal cancer patients after preoperative chemoradiation. Cancer 2008;113:57-64.

26 Abdul-Jalil KI, Sheehan KM, Kehoe J, Cummins R, O'Grady A, McNamara DA, Deasy J, Breathnach O, Grogan L, O’Neill BD, Faul C, Parker I, Kay EW, Hennessy BT, Gillen P: The prognostic value of tumour regression grade following neoadjuvant chemoradiation therapy for rectal cancer. Colorectal Dis 2014;16: 16-25.

27 Hermanek P, Merkel S, Hohenberger W: Prognosis of rectal carcinoma after multimodal treatment: ypTNM classification and tumor regression grading are essential. Anticancer Res 2013;33:559-566.

28 Agarwal A, Chang GJ, Hu CY, Taggart M, Rashid A, Park IJ, You YN, Das P, Krishnan $\mathrm{S}$, Crane $\mathrm{CH}$, Rodriguez-Bigas M, Skibber J, Ellis L, Eng C, Kopetz S, Maru DM: Quantified pathologic response assessed as residual tumor burden is a predictor of recurrencefree survival in patients with rectal cancer who undergo resection after neoadjuvant chemoradiotherapy. Cancer 2013;119:42314241.

29 Kim SH, Chang HJ, Kim DY, Park JW, Baek JY, Kim SY, Park SC, Oh JH, Yu A, Nam BH:
What is the ideal tumor regression grading system in rectal cancer patients after preoperative chemoradiotherapy? Cancer Res Treat 2016;48:998-1009.

30 Cui J, Yang L, Guo L, Shao Y, Tan D, Li N, Zhang H: The combination of early treatment response and $\mathrm{ypT}$ stage is a novel metric to stage rectal cancer patients treated with neoadjuvant chemoradiotherapy. Oncotarget 2017;8:37845-37854.

31 Beddy D, Hyland JM, Winter DC, Lim C, White A, Moriarty M, Armstrong J, Fennelly D, Gibbons D, Sheahan K: A simplified tumor regression grade correlates with survival in locally advanced rectal carcinoma treated with neoadjuvant chemoradiotherapy. Ann Surg Oncol 2008; 15:3471-3477.

32 Maas M, Nelemans PJ, Valentini V, Crane CH, Capirci C, Rodel C, Nash GM, Kuo LJ, Glynne-Jones R, Garcia-Aguilar J, Suarez J, Calvo FA, Pucciarelli S, Biondo S, Theodoropoulos G, Lambregts DM, Beets-Tan RG, Beets GL: Adjuvant chemotherapy in rectal cancer: defining subgroups who may benefit after neoadjuvant chemoradiation and resection: a pooled analysis of 3,313 patients. Int J Cancer 2015;137:212-220.

33 Shahab D, Gabriel E, Attwood K, Ma WW, Francescutti V, Nurkin S, Boland PM: Adjuvant chemotherapy is associated with improved overall survival in locally advanced rectal cancer after achievement of a pathologic complete response to chemoradiation. Clin Colorectal Cancer, Epub ahead of print. 